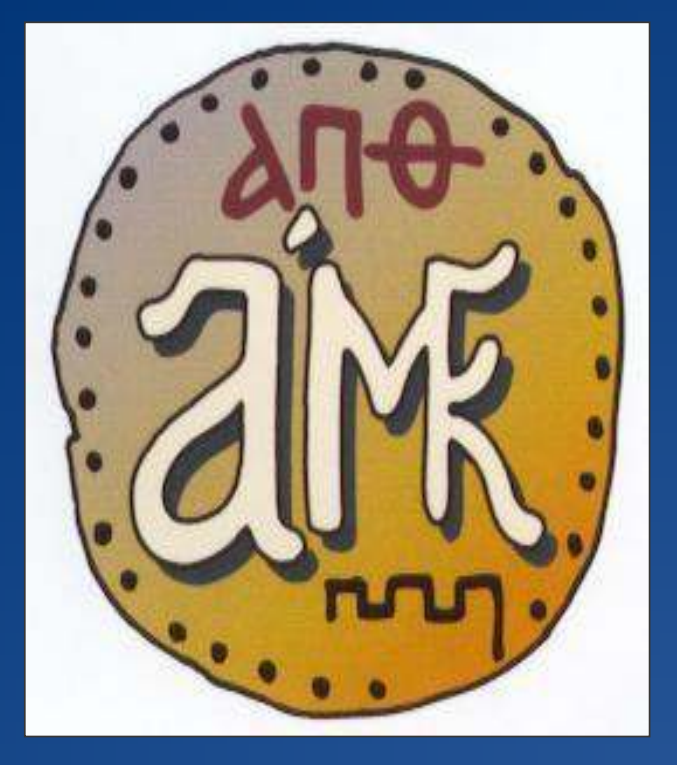

\title{
Primary ovarian small cell carcinoma of
} pulmonary type with enlarged paraaortic lymph node masses: a case report

\section{Tsolakidis D, Klonos E, Papanikolaou E, Ktenidis K., Pervana S Grimbizis GF ,}

First Department of Obstetrics \& Gyneacology "Papageorgiou" General Hospital of Thessaloniki,.

First Surgical Clinic "Papageorgiou General Hospital,

Pathology Department 'Papageorgiou General Hospital Aristotle University of Thessaloniki.

\section{Small cell carcinoma of the ovary of pulmonary type, is a rare, aggressive tumor with poor prognosis and its optimal management is unclear. \\ Case report}

A 55 - year - old Caucasian woman presented with abdominal discomfort and left lumbar pain within a three - week period. At exploratory laparotomy, a $8 \mathrm{~cm}$ solid cystic mass of the left ovary

was found infiltrating the sigmoid colon, and a bulky mass

$(11 \times 7 \times 4 \mathrm{~cm})$ in the left paraaortic infrarenal region. Histopathological features resembling small cell carcinoma of the lungs and positive immunohistochemical stains provided a define diagnosis of IIIC ovarian small cell carcinoma of pulmonary type. After six cycles chemotherapy with carboplatin and etoposide, the patient is still alive at 75 months (6 years and 3 months) from initial diagnosis.

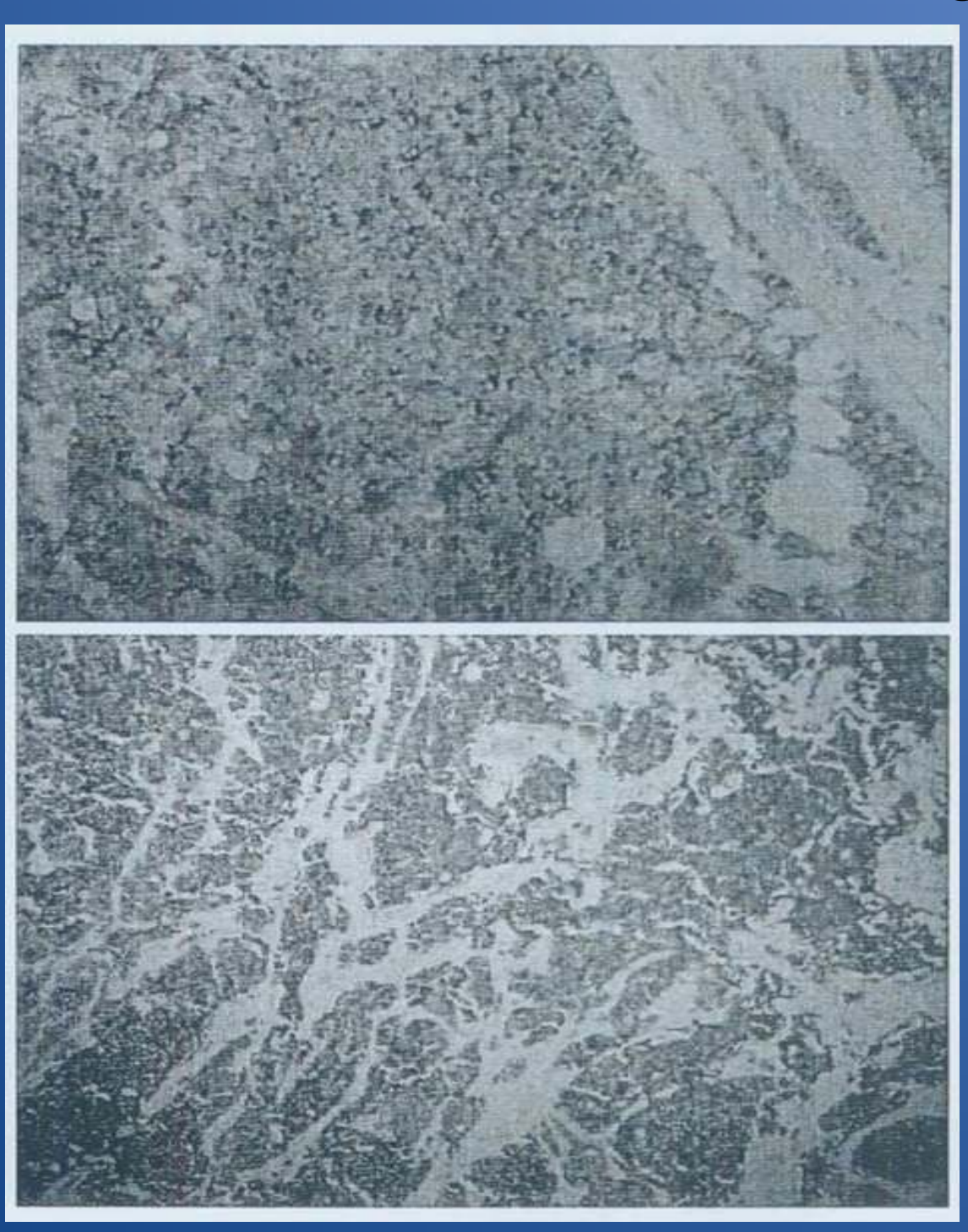

$1^{\text {st }}$ Fig:Immunohistochemical staining for neuron specific enolase which diffusely expressed or expressed as diffuse

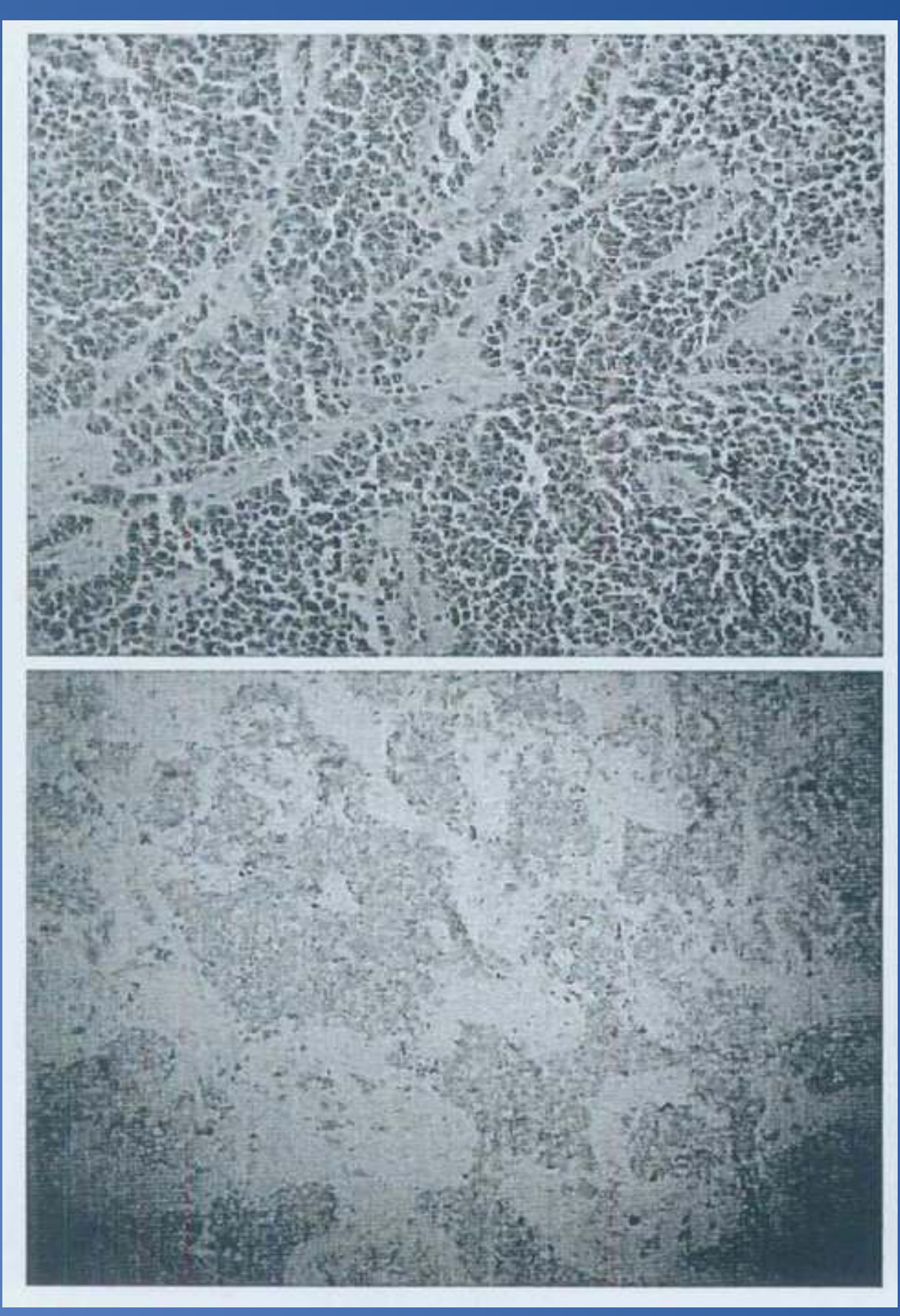

1st Fig: The tumor consisting of pure small cells with scanty cytoplasm and numerous mitotic figures $2^{\text {nd }}$ Fig: Immunohistochemical staining for chromogranin is weakly expressed.

Discussion

In this case, the absence of peritoneal involvement and the extensive paraaortic adenopathy is suggestive of a different pattern of spread of this rare tumor.

Optimal treatment seems to be radical primary debulking surgery resulting in no residual disease, maximizing the effect of adjuvant chemotherapy for this biological aggressive tumor. 